Article

\title{
Gold-catalyzed addition reaction between creatinine and isatin: A sustainable and green chemistry approach for the diastereoselective synthesis of 3-substituted-3-hydroxyisatins
}

\author{
K. Parthasarathy a, T. Ponpandian b ${ }^{\mathrm{b}}$ C. Praveen ${ }^{\mathrm{c}, *}$ \\ a Department of Chemistry, Siddha Central Research Institute, Central Council for Research in Siddha (CCRS), Arumbakkam, Chennai-600106, Tamil Nadu, \\ India \\ b Inogent Laboratories Private Limited, Industrial Development Area Nacharam, Hyderabad-500076, Telangana, India \\ c Functional Materials Division, Council of Scientific and Industrial Research-Central Electrochemical Research Institute (CSIR-CECRI), Karaikudi-600006, \\ Tamil Nadu, India
}

\section{A R T I C L E I N F O}

\section{Article history:}

Received 5 January 2017

Accepted 28 February 2017

Published 5 May 2017

\section{Keywords:}

Creatinine

Gold catalysis

Green chemistry

Diastereoselectivity

Antioxidant

\begin{abstract}
A B S T R A C T
The aldolization of various isatins with creatinine under gold catalysis in water has been developed. The reaction is operationally simple as the products can be isolated by simple filtration without requiring tedious solvent extraction and column chromatographic techniques. The generality of this methodology is showcased through the reactions of a wide range of isatin derivatives with creatinine to afford the respective aldol products in excellent yields with complete syn-selectivity. The scope of this chemistry is further extended to a tandem reaction involving isatins, creatinine and malononitrile to afford multicomponent products in excellent yields with complete anti-selectivity. The antioxidant potency of the synthesized compound was assessed by a spectrophotometric method, which revealed that three compounds containing halogen atoms (2c, $2 \mathbf{d}$ and $\mathbf{2 e}$ ) were the most active compared with the standard.
\end{abstract}

(C) 2017, Dalian Institute of Chemical Physics, Chinese Academy of Sciences. Published by Elsevier B.V. All rights reserved.

\section{Introduction}

The structural motif of 3-substituted-3-hydroxyisatin is present in several bioactive natural products as well as clinical drugs such as paratunamide A, CPC-1 and sporidesmin (Fig. 1) [1-3]. The medicinal properties of these compounds are derived from the $\mathrm{C} 3$ substituent and the absolute configuration of the chiral center [4]. The development of efficient and practical methods to prepare such compounds is of paramount importance and it is an active area of research in asymmetric catalysis [5-7]. One of the simplest preparative procedures for 3-substituted-3-hydroxyisatins is the catalytic addition of nucleophiles to readily available isatins, which grants access to appealing molecular scaffolds possessing quaternary carbon centers $[8,9]$. Furthermore, organic reactions employing water as a medium hold great promise from a green chemistry perspective [10]. For example, Dash et al. [11,12] have previously reported the water-catalyzed diastereoselective aldol reaction of thiazolidinediones with isatin and other aldehydes. As part of our ongoing interest in developing new methodologies for the synthesis of heterocycles [13-24], coupled with the reality that creatinine is present in numerous natural products [25], we envisaged the replacement of thiazolidinediones with structurally relevant creatinine in the aldolization of isatins. In 2010, Crooks et al. [26-29] reported the diastereoselective aldol addition of isatins with creatinine. However, this methodology

* Corresponding author. Tel: +91-9677733808; E-mail: chandrasekar.praveen@gmail.com DOI: 10.1016/S1872-2067(17)62812-2| http://www.sciencedirect.com/science/journal/18722067 | Chin. J. Catal., Vol. 38, No. 5, May 2017 


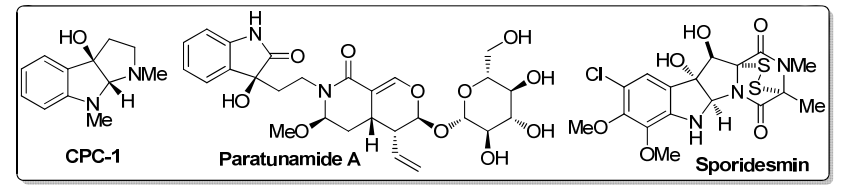

Fig. 1. Natural products containing a 3-hydroxyisatin scaffold.

suffers from the need to use $\mathrm{NaOOCH}_{3}(\mathrm{NaOAc})$ and $\mathrm{CH}_{3} \mathrm{COOH}$ $(\mathrm{AcOH})$ in stoichiometric quantities, which prompted us to revisit this transformation with particular emphasis on performing the reaction under gold catalysis in water without compromising the diastereoselectivity. Herein, we report our study on the gold(III)-catalyzed diastereoselective aldol addition of isatins with creatinine under aqueous condition leading to 3-hydroxyisatin derivatives. In view of the extensive biological properties of 3-hydroxyisatins, all the compounds were screened for their free radical scavenging activity. The gold-catalyzed protocol was also extended to a three-component reaction between isatins, malononitrile and creatinine through a tandem condensation / conjugate addition.

\section{Experimental}

\subsection{Materials, methods and instruments}

Solvents and reagents were purchased from SRL chemicals, India Pvt. Ltd, India and were used without further purification. Melting points (m.p.) were determined in open capillary tubes and are uncorrected. Infrared (IR) spectra were recorded on a Jasco FT-IR spectrophotometer as $\mathrm{KBr}$ pellets. ${ }^{1} \mathrm{H}$ and ${ }^{13} \mathrm{C}$ NMR spectra were obtained in $\mathrm{CDCl}_{3}$ or DMSO- $d_{6}$ solutions on a Bruker spectrometer at 400 and $100 \mathrm{MHz}$, respectively. The proton chemical shifts $(\delta)$ are relative to tetramethylsilane (TMS, $\delta=0.00$ ) as internal standard and are expressed in parts per million (ppm). The spin multiplicities are given as s (singlet), d (doublet), t (triplet) and $m$ (multiplet). The coupling constants () are given in hertz. Mass spectra were recorded on a PE-SCIEX API 3000 mass spectrometer. Elemental analyses were recorded using a ThermoFinnigan FLASH EA 1112CHN analyzer. All compounds gave $\mathrm{C}, \mathrm{H}$ and $\mathrm{N}$ analysis within $\pm 0.5 \%$ of the theoretical values. Analytical TLC was performed on precoated plastic sheets of silica gel G/UV-254 of $0.2 \mathrm{~mm}$ thickness (Macherey-Nagel, Germany) using analytical grade solvents and visualized with iodine spray $\left(10 \%(w / w) \mathrm{I}_{2}\right.$ in silica gel) or UV light ( $\lambda=254$ and $365 \mathrm{~nm}$ ). The absorbance was measured at $517 \mathrm{~nm}$ using a Systronics 118 model spectrophotometer.

\subsection{General procedure for the synthesis of compounds $\mathbf{2 a - 2 p}$}

Water $(15 \mathrm{~mL})$ was added to a mixture of $1.0 \mathrm{mmol}$ of isatin derivative, $1.2 \mathrm{mmol}$ of creatinine (for $\mathbf{2 p}, 2.3 \mathrm{mmol}$ of creatinine) and $1 \mathrm{~mol} \%$ of $\mathrm{HAuCl}_{4}$ and the resulting suspension was heated to reflux for $30 \mathrm{~min}$. The clear reaction mixture was cooled to $15-20^{\circ} \mathrm{C}$. The precipitated aldol product was filtered and washed with copious amount of water and then with methanol and ethyl acetate (EtOAc). The obtained product was thoroughly dried under vacuum to afford the pure product 2a-2p.

3-Hydroxy-3-(2-imino-3-methyl-5-oxoimidazolidin-4-yl)indolin-2-one (2a): Yellow solid; m.p. $=225-227^{\circ} \mathrm{C}$; IR (KBr): $3557,3384,3242,2795,1718,1689,1672,1242,755 \mathrm{~cm}^{-1} .{ }^{1} \mathrm{H}$ NMR (DMSO- $\left.d_{6}\right): \delta 3.14$ (s, 3H, $\left.-\mathrm{CH}_{3}\right), 4.04(\mathrm{~s}, 1 \mathrm{H}, \mathrm{CH}), 6.31$ (brs, $1 \mathrm{H}, \mathrm{OH}), 6.70-6.74\left(\mathrm{~d}, J=7.6 \mathrm{~Hz}, 1 \mathrm{H},-\mathrm{C}_{7} \mathrm{H}\right), 6.81-6.88(\mathrm{t}, J=7.6$ $\left.\mathrm{Hz}, 1 \mathrm{H},-\mathrm{C}_{6} \mathrm{H}\right), 7.06-7.07\left(\mathrm{~d}, J=7.6 \mathrm{~Hz}, 1 \mathrm{H},-\mathrm{C}_{4} \mathrm{H}\right), 7.17-7.20(\mathrm{t}, J$ $=7.8 \mathrm{~Hz}, 1 \mathrm{H},-\mathrm{C}_{5} \mathrm{H}$ ), 7.51 (brs, $2 \mathrm{H}, \mathrm{NH}_{2}$ ), 10.23 (brs, $1 \mathrm{H}, \mathrm{NH}$ ); ${ }^{13} \mathrm{C}$ NMR (DMSO- $d_{6}$ ): $\delta 32.6,69.4,76.3,109.5,121.1,123.9,127.9$, 129.3, 142.6, 171.8, 175.7, 182.3. MS (ESI): $m / z=261[\mathrm{M}+\mathrm{H}]^{+}$; Anal. Calcd. for $\mathrm{C}_{12} \mathrm{H}_{12} \mathrm{~N}_{4} \mathrm{O}_{3}$ : C, 55.38\%; $\mathrm{H}, 4.65 \%$; N, $21.53 \%$. Found C, 55.55\%; H, 4.61\%; N, 21.45\%.

5-Fluoro-3-hydroxy-3-(2-imino-3-methyl-5-oxoimidazolidin-4-yl)indolin-2-one (2b): Pale yellow solid; m.p. $=250-252^{\circ} \mathrm{C}$; IR (KBr): 3358, 3174, 3047, 2697, 1731, 1701, 1645, 1435, 806 $\mathrm{cm}^{-1} .{ }^{1} \mathrm{H}$ NMR (DMSO-d $): \delta 3.14\left(\mathrm{~s}, 3 \mathrm{H}, \mathrm{CH}_{3}\right), 4.07(\mathrm{~s}, 1 \mathrm{H}, \mathrm{CH})$, 6.52 (bs, $1 \mathrm{H}, \mathrm{OH}), 6.70-6.75\left(\mathrm{~m}, 1 \mathrm{H},-\mathrm{C}_{7} \mathrm{H}\right), 6.80-6.84$ (dd, $J=$ $\left.8.1 \mathrm{~Hz}, J=2.37 \mathrm{~Hz} 1 \mathrm{H},-\mathrm{C}_{4} \mathrm{H}\right), 6.99-7.06\left(\mathrm{~m}, 1 \mathrm{H},-\mathrm{C}_{6} \mathrm{H}\right), 7.51$ (brs, $2 \mathrm{H}, \mathrm{NH}_{2}$ ), 10.28 (brs, $1 \mathrm{H}, \mathrm{NH}$ ); ${ }^{13} \mathrm{C}$ NMR (DMSO- $d_{6}$ ): $\delta 32.2,69.8$, 76.5, 111.0, 124.2, 126.0, 129.9, 130.4, 141.9, 172.7, 175.8, 182.1. MS (ESI): $m / z=279[\mathrm{M}+\mathrm{H}]^{+}$; Anal. Calcd. for $\mathrm{C}_{12} \mathrm{H}_{11} \mathrm{FN}_{4} \mathrm{O}_{3}$ : C, $51.80 \% ; \mathrm{H}, 3.98 \%$; N, 20.14\%. Found $\mathrm{C}$, $52.09 \%$; H, 3.92\%; N, 20.05\%.

5-Chloro-3-hydroxy-3-(2-imino-3-methyl-5-oxoimidazolidin-4-yl)indolin-2-one (2c): Pale brown solid; m.p. = 267-269 ${ }^{\circ} \mathrm{C}$; IR (KBr): 3384, 3177, 2782, 2672, 1731, 1707, 1618, 1586, $1083,818 \mathrm{~cm}^{-1}$; ${ }^{1} \mathrm{H}$ NMR (DMSO- $\left.d_{6}\right): \delta 3.16\left(\mathrm{~s}, 3 \mathrm{H}, \mathrm{CH}_{3}\right), 4.07$ (s, $1 \mathrm{H}, \mathrm{CH}), 6.52$ (brs, 1H, OH), 6.74-6.77 (d, J = 8.1 Hz, 1H, $\left.-\mathrm{C}_{7} \mathrm{H}\right)$, 6.99-7.00 (d, $\left.J=2.4 \mathrm{~Hz}, 1 \mathrm{H},-\mathrm{C}_{4} \mathrm{H}\right), 7.23-7.24(\mathrm{dd}, J=8.1 \mathrm{~Hz}, J=$ $2.1 \mathrm{~Hz}, 1 \mathrm{H},-\mathrm{C}_{6} \mathrm{H}$ ), 7.55 (brs, $2 \mathrm{H}, \mathrm{NH}_{2}$ ), 10.39 (brs, $1 \mathrm{H}, \mathrm{NH}$ ); ${ }^{13} \mathrm{C}$ NMR (DMSO-d6): $\delta 32.8,69.6,76.3,110.9,123.9,125.0,129.1$, 129.9, 141.6, 172.0, 175.3, 182.1. MS (ESI): $m / z=295$ $[\mathrm{M}+\mathrm{H}]^{+}, 297[\mathrm{M}+\mathrm{H}]^{2+}$; Anal. Calcd. for $\mathrm{C}_{12} \mathrm{H}_{11} \mathrm{ClN}_{4} \mathrm{O}_{3}: \mathrm{C}, 48.91 \%$; $\mathrm{H}, 3.76 \%$; N 19.01\%. Found C, 49.05\%; H, 3.72\%; N, 18.94\%.

5-Bromo-3-hydroxy-3-(2-imino-3-methyl-5-oxoimidazolidin-4-yl)indolin-2-one (2d): Brown solid; m.p. = 246-248 ${ }^{\circ} \mathrm{C}$; IR (KBr): 3384, 3176, 2980, 2672, 1731, 1707, 1566, 1186, 818 $\mathrm{cm}^{-1} ;{ }^{1} \mathrm{H}$ NMR (DMSO- $\left.d_{6}\right): \delta 3.16\left(\mathrm{~s}, 3 \mathrm{H}, \mathrm{CH}_{3}\right), 4.07(\mathrm{~s}, 1 \mathrm{H}, \mathrm{CH}$ ), 6.53 (brs, 1H, OH), 6.70-6.73 (d, $\left.J=8.1 \mathrm{~Hz}, 1 \mathrm{H},-\mathrm{C}_{6} \mathrm{H}\right), 7.11$ (s, $1 \mathrm{H},-\mathrm{C}_{4} \mathrm{H}$ ), 7.36-7.38 (dd, $J=8.1 \mathrm{~Hz}, J=1.5 \mathrm{~Hz}, 1 \mathrm{H},-\mathrm{C}_{7} \mathrm{H}$ ), 7.76 (brs, $2 \mathrm{H}, \mathrm{NH}_{2}$ ), 10.42 (brs, $1 \mathrm{H}, \mathrm{NH}$ ); ${ }^{13} \mathrm{C}$ NMR (DMSO-d $)$ ): $\delta 32.9$, 69.6, 76.4, 111.5, 112.7, 126.6, 130.4, 132.0, 142.1, 172.1, 175.2, 182.1. MS (ESI): $m / z=339[\mathrm{M}+\mathrm{H}]^{+}, 241[\mathrm{M}+\mathrm{H}]^{2+}$; Anal. Calcd. for $\mathrm{C}_{12} \mathrm{H}_{11} \mathrm{Br}_{4} \mathrm{O}_{3}$ : C, 42.50\%; $\mathrm{H}, 3.27 \%$;, $16.52 \%$. Found $\mathrm{C}$, 42.35\%; H, 3.33\%; N, 16.60\%.

5-Iodo-3-hydroxy-3-(2-imino-3-methyl-5-oxoimidazolidin4-yl)indolin-2-one (2e): Dark brown solid; m.p. = 202-204 ${ }^{\circ} \mathrm{C}$; IR (KBr): 3394, 3176, 2973, 2768, 1730, 1707, 1583, 1308, 1184, $817 \mathrm{~cm}^{-1}$; ${ }^{1} \mathrm{H}$ NMR (DMSO-d6): $\delta 3.17$ (s, 3H, CH 3 ), 4.06 (s, 1H, CH). 6.44 (brs, $1 \mathrm{H}, \mathrm{OH}), 6.66-6.71\left(\mathrm{~d}, J=8.4 \mathrm{~Hz}, 1 \mathrm{H},-\mathrm{C}_{6} \mathrm{H}\right)$, 7.09 (s, $\left.1 \mathrm{H},-\mathrm{C}_{4} \mathrm{H}\right), 7.37-7.40(\mathrm{dd}, J=8.2 \mathrm{~Hz} ; J=1.4 \mathrm{~Hz}, 1 \mathrm{H}$, $\left.-\mathrm{C}_{7} \mathrm{H}\right), 7.13\left(\mathrm{~s}, 1 \mathrm{H},-\mathrm{C}_{4} \mathrm{H}\right), 7.44-7.49(\mathrm{dd}, J=8.4 \mathrm{~Hz}, J=1.6 \mathrm{~Hz}$, $1 \mathrm{H},-\mathrm{C}_{7} \mathrm{H}$ ); ${ }^{13} \mathrm{C}$ NMR (DMSO- $d_{6}$ ): $\delta 33.0,69.8,76.5,111.5,112.8$, $126.9,130.4,132.2,142.3,172.5,175.5,182.6 . \mathrm{MS}$ (ESI): $m / z=$ $387[\mathrm{M}+\mathrm{H}]^{+}$; Anal. Calcd. for $\mathrm{C}_{12} \mathrm{H}_{11} \mathrm{IN}_{4} \mathrm{O}_{3}$ : C, $37.32 \%$; $\mathrm{H}, 2.87 \%$; 
N, 14.51\%. Found C, 37.51\%; H, 2.81\%; N, 14.44\%.

5-Nitro-3-hydroxy-3-(2-imino-3-methyl-5-oxoimidazolidin4-yl)indolin-2-one (2f): Dark yellow solid; m.p. > $300{ }^{\circ} \mathrm{C}$; IR (KBr): 3421, 3345, 3190, 2922, 1734, 1707, 1583, 1313, 1207, $846 \mathrm{~cm}^{-1}$; ${ }^{1} \mathrm{H}$ NMR (DMSO- $\left.d_{6}\right): \delta 3.23\left(\mathrm{~s}, 3 \mathrm{H}, \mathrm{CH}_{3}\right), 4.15(\mathrm{~s}, 1 \mathrm{H}$, $\mathrm{CH}), 6.74(\mathrm{~s}, 1 \mathrm{H}, \mathrm{OH}), 6.95-6.98\left(\mathrm{~d}, J=8.7 \mathrm{~Hz}, 1 \mathrm{H},-\mathrm{C}_{7} \mathrm{H}\right), 7.67$ (bs, $2 \mathrm{H}, \mathrm{NH}_{2}$ ), 7.83-7.84 (d, $J=2.7 \mathrm{~Hz}, 1 \mathrm{H},-\mathrm{C}_{4} \mathrm{H}$ ), 7.81-7.82 (dd, $\left.J=8.7 \mathrm{~Hz}, J=2.4 \mathrm{~Hz}, 1 \mathrm{H},-\mathrm{C}_{7} \mathrm{H}\right), 11.04(\mathrm{~s}, 1 \mathrm{H}, \mathrm{NH}) ;{ }^{13} \mathrm{C} \mathrm{NMR}$ (DMSO-d6): $\delta 33.1,69.9,75.8,109.8,119.2,126.9,128.9,141.5$, 149.4, 172.3, 176.0, 181.9. MS (ESI): $m / z=305[\mathrm{M}+\mathrm{H}]^{+}$; Anal. Calcd. for $\mathrm{C}_{12} \mathrm{H}_{12} \mathrm{~N}_{5} \mathrm{O}_{5}$ : C, 47.22\%; $\mathrm{H}, 3.63 \%$;, $22.94 \%$. Found C, $46.99 \%$; H, 3.68\%; N, 23.04\%.

3-Hydroxy-3-(2-imino-3-methyl-5-oxoimidazolidin-4-yl)-1methylindolin-2-one (2g): Pale yellow solid; m.p. $=230-232{ }^{\circ} \mathrm{C}$; IR (KBr): 3603, 3384, 3174, 2884, 1722, 1711, 1648, 1102, 754 $\mathrm{cm}^{-1}$; ${ }^{1} \mathrm{H}$ NMR (DMSO-d $): \delta 3.05\left(\mathrm{~s}, 3 \mathrm{H}, \mathrm{CH}_{3}\right), 3.18(\mathrm{~s}, 3 \mathrm{H}$, $\left.\mathrm{CH}_{3}\right), 4.10(\mathrm{~s}, 1 \mathrm{H}, \mathrm{CH}), 6.45$ (brs, $\left.1 \mathrm{H}, \mathrm{OH}\right), 6.91-6.97(\mathrm{~m}, 2 \mathrm{H}$, $-\mathrm{C}_{5} \mathrm{H},-\mathrm{C}_{6} \mathrm{H}$ ), 7.07-7.09 (d, $\left.J=7.5 \mathrm{~Hz}, 1 \mathrm{H}, \mathrm{C}_{7} \mathrm{H}\right), 7.25-7.31(\mathrm{t}, J=$ $8.1 \mathrm{~Hz}, 1 \mathrm{H},-\mathrm{C}_{4} \mathrm{H}$ ), 7.55 (brs, $2 \mathrm{H}, \mathrm{NH}_{2}$ ); ${ }^{13} \mathrm{C}$ NMR (DMSO- $d_{6}$ ): $\delta$ 25.9, 32.6, 69.9, 76.3, 109.2, 111.2, 115.9, 129.0, 140.4, 156.4, 171.9, 174.0, 181.9. MS (ESI): $m / z=275[\mathrm{M}+\mathrm{H}]^{+}$; Anal. Calcd. for $\mathrm{C}_{13} \mathrm{H}_{14} \mathrm{~N}_{4} \mathrm{O}_{3}$ : C, 56.93\%; $\mathrm{H}, 5.14 \%$;, $20.43 \%$. Found $\mathrm{C}$, $57.08 \%$; H, 5.09\%, N, 19.99\%.

3-Hydroxy-3-(2-imino-3-methyl-5-oxoimidazolidin-4-yl)-1ethylindolin-2-one (2h): Pale yellow solid; m.p. = 196-198 ${ }^{\circ} \mathrm{C}$; IR (KBr): 3603, 3381, 3176, 2985, 1726, 1710, 1698, 1338, 1102, $754 \mathrm{~cm}^{-1}$; ${ }^{1} \mathrm{H}$ NMR (DMSO-d6): $\delta 1.32(\mathrm{t}, J=7.6 \mathrm{~Hz}, 3 \mathrm{H}$, $\mathrm{CH}_{2}$ ), 3.12 (s, 3H, CH 3$), 3.22\left(\mathrm{~s}, 3 \mathrm{H}, \mathrm{CH}_{3}\right), 4.15(\mathrm{~s}, 1 \mathrm{H}, \mathrm{CH}), 6.52$ (brs, $1 \mathrm{H}, \mathrm{OH}), 6.88-6.94\left(\mathrm{~m}, 2 \mathrm{H},-\mathrm{C}_{5} \mathrm{H},-\mathrm{C}_{6} \mathrm{H}\right), 7.07-7.09(\mathrm{~d}, J=$ $7.6 \mathrm{~Hz}, 1 \mathrm{H}, \mathrm{C}_{7} \mathrm{H}$ ), 7.27-7.33 (t, $J=8.1 \mathrm{~Hz}, 1 \mathrm{H},-\mathrm{C}_{4} \mathrm{H}$ ), 7.64 (brs, $2 \mathrm{H}, \mathrm{NH}_{2}$ ); ${ }^{13} \mathrm{C}$ NMR (DMSO-d $): \delta 19.1,25.9,32.9,70.5,76.3$, 109.7, 111.9, 115.4, 129.0, 140.8, 156.8, 172.3, 174.5, 182.5. MS (ESI): $m / z=289[\mathrm{M}+\mathrm{H}]+$; Anal. Calcd. for $\mathrm{C}_{14} \mathrm{H}_{16} \mathrm{~N}_{4} \mathrm{O}_{3}: \mathrm{C}$, $58.32 \%$; H, 5.59\%; N, 19.43\%. Found C, 57.99\%; H, 5.65\%; N, $19.60 \%$

3-Hydroxy-3-(2-imino-3-methyl-5-oxoimidazolidin-4-yl)-1hexylindolin-2-one (2i): Pale yellow solid; m.p. $=183-185^{\circ} \mathrm{C}$; IR (KBr): 3366, 3314, 3173, 3063, 2956, 1696, 1652, 1498, 763 $\mathrm{cm}^{-1}$; ${ }^{1} \mathrm{H}$ NMR (DMSO- $d_{6}$ ): $\delta 0.87\left(\mathrm{t}, J=7.6 \mathrm{~Hz}, 3 \mathrm{H},-\mathrm{CH}_{3}\right.$ ), $1.22-1.35\left(\mathrm{~m}, 6 \mathrm{H},-\left(\mathrm{CH}_{2}\right)_{3}-\mathrm{Me}\right), 1.40-1.44\left(\mathrm{~m}, 2 \mathrm{H},-\mathrm{CH}_{2}-\right) ; 3.25$ (s, 3H, NMe), $3.44(\mathrm{t}, J=7.6 \mathrm{~Hz}, 2 \mathrm{H}) ; 4.22(\mathrm{~s}, 1 \mathrm{H}, \mathrm{CH}), 5.99$ (brs, $1 \mathrm{H}, \mathrm{OH}), 6.95(\mathrm{~d}, J=8.4 \mathrm{~Hz}, 1 \mathrm{H}, \mathrm{ArH}) ; 7.19-7.24(\mathrm{~m}, 2 \mathrm{H}, \mathrm{ArH})$, 7.36-7.41 (m, 1H, ArH) 7.77 (brs, 2H, $\left.-\mathrm{NH}_{2}\right) .{ }^{13} \mathrm{C}$ NMR (DMSO-d $)$ ): $\delta 13.9,21.9,26.5,27.2,28.1,31.4,44.4,77.7,88.4$, 115.4, 125.2, 125.7, 127.7, 128.9, 144.5, 159.2, 174.1, 179.9. MS (ESI): $m / z=345[\mathrm{M}+\mathrm{H}]+$; Anal. Calcd. for $\mathrm{C}_{18} \mathrm{H}_{24} \mathrm{~N}_{4} \mathrm{O}_{3}: \mathrm{C}$, 62.77\%; H, 7.02\%; N, 16.27\%. Found C, 63.01\%; H, 6.96\%; N, $16.20 \%$.

3-Hydroxy-3-(2-imino-3-methyl-5-oxoimidazolidin-4-yl)-1phenylindolin-2-one (2j): Pale yellow solid; m.p. $=229-231^{\circ} \mathrm{C}$; IR (KBr): 3402, 3199, 2948, 1722, 1700, 1132, $843 \mathrm{~cm}^{-1}$; ${ }^{1} \mathrm{H}$ NMR (DMSO- $d_{6}$ ): $\delta 3.25$ (s, 3H, CH $), 4.22$ (s, 1H, CH), 6.62 (brs, $1 \mathrm{H}, \mathrm{OH}), 6.64-6.65\left(\mathrm{~d}, J=0.6 \mathrm{~Hz}, 1 \mathrm{H}, \mathrm{C}_{4} \mathrm{H}\right), 6.98-7.03(\mathrm{t}, J=7.6$ $\mathrm{Hz}, 1 \mathrm{H}, \mathrm{Ar}-\mathrm{H}$ ), 7.17-7.23 (m, 2H, $\left.\mathrm{C}_{5} \mathrm{H}, \mathrm{C}_{6} \mathrm{H}\right), 7.44-7.60$ (m, 5H, $\mathrm{Ar}-\mathrm{H}, \mathrm{C}_{7} \mathrm{H}$ ), 7.70 (brs, $2 \mathrm{H}, \mathrm{NH}_{2}$ ); ${ }^{13} \mathrm{C}$ NMR (DMSO- $d_{6}$ ): $\delta 33.0$, 70.6, 76.3, 108.9, 122.7, 124.4, 126.9, 127.1, 127.2, 128.1, 129.6, 129.7, 129.9, 134.4, 143.9, 171.9, 174.1, 182.5. MS (ESI): $m / z=$
$337[\mathrm{M}+\mathrm{H}]^{+}$; Anal. Calcd. for $\mathrm{C}_{18} \mathrm{H}_{16} \mathrm{~N}_{4} \mathrm{O}$ : C, 64.28\%; $\mathrm{H}, 4.79 \%$; $16.66 \%$. Found C, 63.99\%; H, 4.71\%; N, 16.57\%.

1-Benzyl-3-hydroxy-3-(2-imino-3-methyl-5-oxoimidazolidin-4-yl)indolin-2-one (2k): Pale brown solid; m.p. = 207-209 ${ }^{\circ} \mathrm{C}$; IR (KBr): 3377, 3312, 3199, 3065, 3031, 2824, 1705, 1644, 1575, 1215, $798 \mathrm{~cm}^{-1}$; ${ }^{1} \mathrm{H}$ NMR (DMSO-d6): $\delta 3.20$ (s, 3H, NMe), 4.04 (s, 1H, CH), 5.22 (s, 2H, $-\mathrm{CH}_{2} \mathrm{Ph}$ ), 6.21 (brs, 1H, OH), 6.93 (d, $J=7.6 \mathrm{~Hz}, 1 \mathrm{H}, \mathrm{ArH}$ ), 7.19-7.27 (m, $5 \mathrm{H}, \mathrm{ArH}), 7.34-7.39$ (m, 3H), 7.69 (brs, $2 \mathrm{H}, \mathrm{NH}_{2}$ ). ${ }^{13} \mathrm{C}$ NMR (DMSO-d6): $\delta 35.1,55.5,83.3$, 94.1, 118.1, 124.7, 126.0, 127.0, 127.9, 128.4, 128.5, 147.5, 171.2, 175.1, 180.9. MS (ESI): $m / z=351[\mathrm{M}+\mathrm{H}]^{+}$; Anal. Calcd. for $\mathrm{C}_{19} \mathrm{H}_{18} \mathrm{~N}_{4} \mathrm{O}_{3}$ : C, $65.13 \% ; \mathrm{H}, 5.18 \%$;, $15.99 \%$. Found $\mathrm{C}$. 64.95\%; H, 5.25\%; N, 16.11\%.

1-Acetyl-3-hydroxy-3-(2-imino-3-methyl-5-oxoimidazolidin-4-yl)indolin-2-one (2l): Colorless solid; m.p. = $175-177^{\circ} \mathrm{C}$; IR (KBr): 3603, 3381, 3175, 2985, 2939, 1727, 1710, 1698, 1649, 1375, $754 \mathrm{~cm}^{-1} ;{ }^{1} \mathrm{H}$ NMR (DMSO- $\left.d_{6}\right): \delta 2.58(\mathrm{~s}, 3 \mathrm{H}$, $\left.-\mathrm{COCH}_{3}\right), 3.23$ (s, 3H, $\left.\mathrm{CH}_{3}\right), 4.24(\mathrm{~s}, 1 \mathrm{H}, \mathrm{CH}), 6.77$ (brs, $\left.1 \mathrm{H}, \mathrm{OH}\right)$, 7.17-7.38 (m, 3H, $\mathrm{C}_{4} \mathrm{H}, \mathrm{C}_{5} \mathrm{H}, \mathrm{C}_{6} \mathrm{H}$ ), 7.46 (brs, 1H, NH), 7.56 (brs, $1 \mathrm{H}, \mathrm{NH}$ ), 8.04-8.06 (d, $J=8.1 \mathrm{~Hz}, 1 \mathrm{H}, \mathrm{C} 7 \mathrm{H}$ ); ${ }^{13} \mathrm{C}$ NMR (DMSO- $d_{6}$ ): $\delta$ 26.1, 32.9, 71.0, 76.3, 117.3, 123.9, 128.7, 129.2, 129.7, 139.1, 169.9, 172.3, 175.4, 181.9. MS (ESI): $m / z=303[\mathrm{M}+\mathrm{H}]^{+}$; Anal. Calcd. for $\mathrm{C}_{14} \mathrm{H}_{14} \mathrm{~N}_{4} \mathrm{O}_{4}$ : C, 55.63\%; H, 4.67\%; N, 18.53\%. Found C, 55.75\%; H, 4.65\%; N, 18.45\%.

1-Benzoyl-3-hydroxy-3-(2-imino-3-methyl-5-oxoimidazolidin-4-yl)indolin-2-one (2m): Colorless solid; m.p. = 224-226 ${ }^{\circ} \mathrm{C}$; IR (KBr): 3600, 3380, 3166, 2989, 2925, 1730, 1709, 1700, $1645,1379,755 \mathrm{~cm}^{-1}$; ${ }^{1} \mathrm{H}$ NMR (DMSO- $d_{6}$ ): $\delta 3.17$ (s, $3 \mathrm{H}, \mathrm{CH}_{3}$ ), 4.21 (s, $1 \mathrm{H}, \mathrm{CH}), 4.74-4.91(\mathrm{ABq}, J=16.2 \mathrm{~Hz}), 6.57$ (brs, $1 \mathrm{H}$, $\mathrm{OH}), 6.64-6.67\left(\mathrm{~d}, J=8.1 \mathrm{HZ}, 1 \mathrm{H},-\mathrm{C}_{4} \mathrm{H}\right), 6.91-6.96(\mathrm{t}, J=7.6 \mathrm{~Hz}$, $\left.1 \mathrm{H},-\mathrm{C}_{5} \mathrm{H}\right), 7.11-7.34\left(\mathrm{~m}, 5 \mathrm{H},-\mathrm{C}_{6} \mathrm{H},-\mathrm{C}_{7} \mathrm{H}\right.$ and $\left.\mathrm{Ar}-\mathrm{H}\right), 7.45-7.47$ (d, $J=7.2 \mathrm{~Hz}, 2 \mathrm{H}, \mathrm{Ar}-\mathrm{H}$ ), 7.56 (brs, $2 \mathrm{H}, \mathrm{NH}_{2}$ ); ${ }^{13} \mathrm{C}$ NMR (DMSO-d6): $\delta 32.7,42.9,69.5,76.0,109.0,121.9,123.7,127.0$, 127.2, 127.3, 127.4, 128.2, 128.3, 129.4, 136.0, 143.2, 171.9, 174.4, 182.3. MS (ESI): $m / z=365[\mathrm{M}+\mathrm{H}]+$; Anal. Calcd. for $\mathrm{C}_{19} \mathrm{H}_{18} \mathrm{~N}_{4} \mathrm{O}_{3}$ : C, 62.63\%; H, 4.43\%; N, 15.38\%. Found C, 62.75\%; $\mathrm{H}, 4.38 \%$; N, $15.30 \%$.

3-Hydroxy-3-(2-imino-3-methyl-5-oxoimidazolidin-4-yl)-1(phenylsulfonyl)indolin-2-one (2n): Colorless solid; m.p. = 230-232 ${ }^{\circ} \mathrm{C}$; IR (KBr): 3444, 3389, 3267, 2968, 1768, 1725, $1701,1682,1544,1108,858 \mathrm{~cm}^{-1}$; ${ }^{1} \mathrm{H}$ NMR (DMSO-d $\left.d_{6}\right): \delta 3.13$ (s, 3H, $\left.\mathrm{CH}_{3}\right), 4.17$ (s, 1H, CH), 6.93 (brs, $\left.1 \mathrm{H}, \mathrm{OH}\right), 7.15-7.17$ (m, $\left.2 \mathrm{H}, \mathrm{C}_{5} \mathrm{H}, \mathrm{C}_{6} \mathrm{H}\right), 7.36-7.42(\mathrm{~m}, 2 \mathrm{H}, \mathrm{Ar}-\mathrm{H}), 7.62-7.84(\mathrm{~m}, 5 \mathrm{H}, \mathrm{Ar}-\mathrm{H}$, $\left.\mathrm{NH}_{2}\right), 8.05-8.08\left(\mathrm{~m}, 2 \mathrm{H}, \mathrm{C}_{4} \mathrm{H}, \mathrm{C}_{7} \mathrm{H}\right) ;{ }^{13} \mathrm{C}$ NMR (DMSO- $\left.d_{6}\right): \delta 33.32$, 70.7, 78.3, 122.4, 124.3, 124.5, 126.9, 127.0, 127.1, 128.3, 128.4, 129.5, 135.4,142.1, 171.9 (C=N), 173.9, 182.6. MS (ESI): $m / z=$ $401[\mathrm{M}+\mathrm{H}]^{+}$; Anal. Calcd. for $\mathrm{C}_{18} \mathrm{H}_{16} \mathrm{~N}_{4} \mathrm{O}_{5} \mathrm{~S}$ : C, 53.99\%; $\mathrm{H}, 4.03 \%$; N, 13.99\%; S, 8.01\%. Found C, 60.11\%; H, 3.99\%; N, 14.07\%; S $7.95 \%$.

5,6-Dibromo-3-hydroxy-3-(2-imino-3-methyl-5-oxoimidazolidin-4-yl)indolin-2-one (2o): Brown solid; m.p. = 196-198 ${ }^{\circ} \mathrm{C}$; IR (KBr): 3391, 3350, 3246, 3176, 2782, 2607, 1731, 1706, 1585, 1477, 1082, $818 \mathrm{~cm}^{-1}$; ${ }^{1} \mathrm{H}$ NMR (DMSO-d6): $\delta 3.17$ (s, 3H, $\mathrm{CH}_{3}$ ), 4.10 (s, 1H, CH), 6.69 (brs, $\left.1 \mathrm{H}, \mathrm{OH}\right), 7.10\left(\mathrm{~s}, 1 \mathrm{H},-\mathrm{C}_{6} \mathrm{H}\right.$ ), 7.52 (brs, 1H, NH), 7.67 (d, $1 \mathrm{H}, J=1.8 \mathrm{~Hz},-\mathrm{C}_{4} \mathrm{H}$ ), 7.85 (bs, $1 \mathrm{H}$, $\mathrm{NH}), 10.80$ (brs, $1 \mathrm{H}, \mathrm{NH}$ ); ${ }^{13} \mathrm{C}$ NMR (DMSO-d $): \delta 32.9,69.8$, 77.0, 102.8, 113.2, 125.7, 131.6, 133.9, 141.7, 172.2, 175.1, 
181.9. MS (ESI): $m / z=417[\mathrm{M}+\mathrm{H}]^{+}, 419[\mathrm{M}+\mathrm{H}]^{2+}, 421[\mathrm{M}+\mathrm{H}]^{4+}$; Anal. Calcd. for $\mathrm{C}_{12} \mathrm{H}_{10} \mathrm{Br}_{2} \mathrm{~N}_{4} \mathrm{O}_{3}$ : C, 34.48\%, $\mathrm{H}, 2.41 \%$, N, $13.40 \%$. Found C, 34.59\%; H, 2.36\%; N, 13.33\%.

1,1'-(Propane-1,3-diyl)bis(3-hydroxy-3-(2-imino-3-methyl5-oxoimidazolidin-4-yl)indolin-2-one (2p): Pale brown solid; m.p. > $300{ }^{\circ} \mathrm{C}$; IR (KBr): 3361, 3189, 1708, 1644, 1467, 1200, $755 \mathrm{~cm}^{-1}$; mp 287-289 ${ }^{\circ} \mathrm{C}$; ${ }^{1} \mathrm{H}$ NMR (DMSO- $\left.d_{6}\right): \delta 1.55-1.61(\mathrm{~m}$, $2 \mathrm{H}), 3.01(\mathrm{~s}, 3 \mathrm{H}, \mathrm{NMe}), 4.02(\mathrm{t}, J=7.6 \mathrm{~Hz}, 4 \mathrm{H}), 4.27(\mathrm{~s}, 2 \mathrm{H}, \mathrm{CH})$, 6.05 (brs, 2H, OH), 7.00 (d, 2H, J = 8.4 Hz, Ar-H), 7.21 (d, 2H, $J=$ $8.4 \mathrm{~Hz}, \mathrm{Ar}-\mathrm{H}$ ), 7.29-7.42 (m, 4H, Ar-H), 7.83 (brs, 2H, NH), 9.89 (brs, 2H, NH). ${ }^{13} \mathrm{C}$ NMR (DMSO- $d_{6}$ ): $\delta 22.9,35.7,39.9,82.1,90.7$, $115,1,124.2,124.8,126.7,127.1,142.6,153.8,174.7,179.9 . \mathrm{MS}$ (ESI): $m / z=561[\mathrm{M}+\mathrm{H}]+$; Anal. Calcd. for $\mathrm{C}_{27} \mathrm{H}_{28} \mathrm{~N}_{8} \mathrm{O}_{6}: \mathrm{C}$, 57.85\%; H, 5.03\%; N, 19.99\%. Found C, 58.04\%; H, 4.99\%; N, $19.82 \%$.

\subsection{General procedure for the synthesis of compounds $(\mathbf{3 a}-\mathbf{3 e})$}

A mixture of $1.0 \mathrm{mmol}$ of isatin, $1.0 \mathrm{mmol}$ of malononitrile and $1 \mathrm{~mol} \%$ of chloroauric acid in $15 \mathrm{~mL}$ of water was stirred at room temperature for $15 \mathrm{~min}$. To this mixture was added 1.2 mmol of creatinine and the resulting suspension was heated to reflux for $30 \mathrm{~min}$ and then cooled to room temperature. The precipitated product was filtered and washed with copious amount of water and then with methanol and ethyl acetate. The obtained product was thoroughly dried under vacuum to afford the crude product. The crude product was purified by column chromatography using hexane/EtOAc eluent system to afford the inseparable diastereoisomeric mixture of $\mathbf{3 a}-\mathbf{3 e}$.

2-(3-(2-Imino-3-methyl-5-oxoimidazolidin-4-yl)-2-oxoindolin-3-yl)malononitrile (3a): Pale yellow solid; m.p. $=201-203$ ${ }^{\circ} \mathrm{C}$; IR (KBr): 3324, 2912, 2255, 1725, 1702, 1305, $877 \mathrm{~cm}^{-1}$; ${ }^{1} \mathrm{H}$ NMR (DMSO-d6): $\delta 2.33$ (s, 3H, NMe), 4.34 (CH-CO), 5.93 (s, 1H, $\left.\mathrm{CH}(\mathrm{CN})_{2}\right), 6.96(\mathrm{~d}, J=6.0 \mathrm{~Hz}, 1 \mathrm{H}), 7.15(\mathrm{~s}, 1 \mathrm{H}, \mathrm{ArH}), 7.40(\mathrm{~s}, 1 \mathrm{H}$, ArH), 7.49 (s, 1H, ArH), 7.50 (brs, 1H, -NH), 8.03 (brs, 1H, -NH), 11.07 (brs, $1 \mathrm{H}, \mathrm{NH}-\mathrm{CO}$ ). ${ }^{13} \mathrm{C}$ NMR (DMSO- $d 6$ ): $\delta 33.2,43.9,53.3$, 66.1, 111.0, 111.6, 112.3, 123.1, 124.5, 125.0, 131.4, 142.8, 172.7, 173.4, 183.1. MS (ESI): $m / z=309[\mathrm{M}+\mathrm{H}]^{+}$; Anal. Calcd. for $\mathrm{C}_{15} \mathrm{H}_{12} \mathrm{~N}_{6} \mathrm{O}_{2}$ : C, $58.44 \% ; \mathrm{H}, 3.92 \% ; \mathrm{N}, 27.26 \%$. Found $\mathrm{C}$, $58.89 \%$; H, 3.85\%; N, 27.10\%.

2-(5-Fluoro-3-(2-imino-3-methyl-5-oxoimidazolidin-4-yl)2-oxoindolin-3-yl)malononitrile (3b): Colorless solid; m.p. = 165-167 ${ }^{\circ} \mathrm{C}$; IR (KBr): 3329, 2922, 2233, 1717, 1632, 1314, 799 $\mathrm{cm}^{-1}$; ${ }^{1} \mathrm{H}$ NMR (DMSO-d $\left.d_{6}\right): \delta 2.50\left(\mathrm{~s}, 3 \mathrm{H}, \mathrm{NCH}_{3}\right.$ ), $4.38(\mathrm{~s}, 1 \mathrm{H}$, $\mathrm{CH}-\mathrm{CO}), 5.95$ (s, 1H, $\left.\mathrm{CH}(\mathrm{CN})_{2}\right), 6.96-6.99(\mathrm{~m}, 1 \mathrm{H}, \mathrm{ArH})$, 7.25-7.32 (m, 2H, ArH), 7.59 (brs, 1H, NH), 8.07 (brs, 1H, NH), 11.11 (brs, $1 \mathrm{H}, \mathrm{NH}-\mathrm{CO}) .{ }^{13} \mathrm{C}$ NMR (DMSO-d $): \delta 33.3,53.6,66.1$, 111.2, 111.8, 112.3, 112.4 ( $\mathrm{C}-\mathrm{F}=32.0 \mathrm{~Hz}), 112.6,118.0,118.2$, 126.0, 139.1, 157.2, 159.6 (UC-F $=152.0 \mathrm{~Hz}), 172.6,173.0,182.7$. MS (ESI): $m / z=327[\mathrm{M}+\mathrm{H}]^{+}$; Anal. Calcd. for $\mathrm{C}_{15} \mathrm{H}_{11} \mathrm{FN}_{6} \mathrm{O}_{2}: \mathrm{C}$, $55.22 \%$; H, 3.40\%; N, 25.76\%. Found C, 54.90\%; H, 3.45\%; N, $25.91 \%$.

2-(5-Chloro-3-(2-imino-3-methyl-5-oxoimidazolidin-4-yl)2-oxoindolin-3-yl)malononitrile (3c): Colorless solid; m.p. = 189-191 ㄷ; IR (KBr): 3325, 2978, 2248, 1728, 1699, 1344, 813 $\mathrm{cm}^{-1}$; ${ }^{1} \mathrm{H}$ NMR (DMSO-d $): \delta 2.62\left(\mathrm{~s}, 3 \mathrm{H}, \mathrm{NCH}_{3}\right.$ ), $4.39(\mathrm{~s}, 1 \mathrm{H}$, CH-CO), $5.98\left(1 \mathrm{H}, \mathrm{s}, \mathrm{CH}(\mathrm{CN})_{2}\right), 6.98(\mathrm{~d}, J=6.9 \mathrm{~Hz}, 1 \mathrm{H}, \mathrm{ArH}), 7.46$ (d, $J=8.3 \mathrm{~Hz}, 2 \mathrm{H}, \mathrm{ArH}$ ), 7.62 (brs, 1H, NH), 8.09 (brs, 1H, NH), 11.22 (brs, $1 \mathrm{H}, \mathrm{NH}-\mathrm{CO}) .{ }^{13} \mathrm{C}$ NMR (DMSO- $d_{6}$ ): $\delta 27.0,53.0,66.0$, 111.0, 111.6, 112.1, 124.3, 126.1, 126.5, 130.9, 141.5, 172.1, 173.2, 182.3. MS (ESI): $m / z=343[\mathrm{M}+\mathrm{H}]^{+}, 345[\mathrm{M}+\mathrm{H}]^{2+}$; Anal. Calcd. for $\mathrm{C}_{15} \mathrm{H}_{11} \mathrm{ClN}_{6} \mathrm{O}_{2}$ : C, 52.56\%; $\mathrm{H}, 3.23 \%$; N, 24.52\%. Found C, $52.90 \%$; H, 3.17\%; N, 24.41\%.

2-(5-Nitro-3-(2-imino-3-methyl-5-oxoimidazolidin-4-yl)-2oxoindolin-3-yl)malononitrile (3d): Dark yellow solid; m.p. = 297-299 C; IR (KBr): 3308, 2951, 2251, 1701, 1689, 1378, 798 $\mathrm{cm}^{-1}$; ${ }^{1} \mathrm{H}$ NMR (DMSO- $\left.d_{6}\right): \delta 2.68\left(\mathrm{~s}, 3 \mathrm{H},-\mathrm{NCH}_{3}\right), 4.48(\mathrm{~s}, 1 \mathrm{H}$, CH-CO), 6.15 (s, 1H, CH(CN)2), 7.19 (d, J = 8.4 Hz, 1H, ArH), 8.16-8.55 (m, 2H, ArH), 9.31 (brs, 1H, NH), 10.17 (brs, 1H, NH), 11.87 (brs, $1 \mathrm{H}, \mathrm{NH}-\mathrm{CO}) .{ }^{13} \mathrm{C}$ NMR (DMSO- $d_{6}$ ): $\delta 34.0,52.7,66.9$, 110.9, 111.5, 120.3, 124.9, 128.1, 142.4, 149.2, 173.0, 174.0, 182.1. MS (ESI): $m / z=354[\mathrm{M}+\mathrm{H}]^{+}$; Anal. Calcd. for $\mathrm{C}_{15} \mathrm{H}_{11} \mathrm{~N}_{7} \mathrm{O}_{4}$ : C, 50.99\%; H, 3.14\%; N, 27.75\%. Found C, 51.22\%; H, 3.08\%; N, $27.60 \%$.

2-(3-(2-Imino-3-methyl-5-oxoimidazolin-4-yl)-5-methyl-2-oxoindolin-3-yl)malononitrile (3e): Light yellow solid; m.p. $=213-215^{\circ} \mathrm{C}$; IR (KBr): 3301, 2934, 2250, 1711, 1678, 1311, $843 \mathrm{~cm}^{-1}$; ${ }^{1} \mathrm{H}$ NMR (DMSO- $\left.d_{6}\right): \delta 2.31\left(\mathrm{~s}, 3 \mathrm{H},-\mathrm{CH}_{3}\right), 2.39(\mathrm{~s}, 3 \mathrm{H}$, $\left.-\mathrm{NCH}_{3}\right), 4.29$ (s, 1H, CH-CO), $5.90\left(\mathrm{~s}, 1 \mathrm{H}, \mathrm{CH}(\mathrm{CN})_{2}\right), 6.86$ (d, J = $7.1 \mathrm{~Hz}, 1 \mathrm{H}, \mathrm{ArH}), 7.21$ (d, J=6.92 Hz, 1H, ArH), 7.33 (s, 1H, ArH), 7.49 (brs, 1H, NH), 8.00 (brs, 1H, NH), 10.95 (brs, 1H, NH-CO). ${ }^{13}$ C NMR (DMSO- $d_{6}$ ): $\delta 21.1,22.3,44.0,53.3,66.0,110.8,111.6$, $112.3,125.0,131.6,132.2,140.3,172.6,173.2,183.2$. MS (ESI): $m / z=323[\mathrm{M}+\mathrm{H}]^{+}$; Anal. Calcd. for $\mathrm{C}_{16} \mathrm{H}_{14} \mathrm{~N}_{6} \mathrm{O}_{2}$ : C, 56.92\%; $\mathrm{H}$, 4.38\%; N, 26.07\%. Found C, 57.05\%; H, 4.32\%; N, 25.99\%.

\subsection{General procedure for the determination of radical scavenging activity of 3-hydroxyisatins $(\mathbf{2 a - 2 o})$ by the DPPH method}

To a $3 \mathrm{~mL}$ ethanolic solution of 1,1-diphenyl-2-picryl-hydrazil (DPPH, $200 \mu \mathrm{mol} / \mathrm{L}$ ), $0.05 \mathrm{~mL}$ of different concentration (50, $500,1000 \mu \mathrm{g} / \mathrm{mL}$ ) of test samples and $20 \mu \mathrm{g}$ of ascorbic acid were added. The solutions were incubated at $37^{\circ} \mathrm{C}$ for $30 \mathrm{~min}$. The absorbance was measured at $517 \mathrm{~nm}$ using a Systronics 118 model spectrophotometer. The percentage inhibition of the DPPH radical was calculated by comparing the results of the test with those of the control using the formula: inhibition $(\%)=\left(A_{\mathrm{C}}-A_{\mathrm{T}}\right) / A_{\mathrm{C}} \times 100$, where $A_{\mathrm{c}}$ is the absorbance of the control sample and $A_{\mathrm{T}}$ is the absorbance of test sample.

\subsection{General procedure for the hydrogen peroxide radical scavenging activity of 3-hydroxyisatins (2a-2o)}

Hydrogen peroxide radical scavenging activity was performed by dissolving $10 \mu \mathrm{g}$ of each 3-hydroxyisatin (2a-2o) in $3.4 \mathrm{~mL}$ of $0.1 \mathrm{~mol} / \mathrm{L}$ phosphate buffer $(\mathrm{pH}=7.4)$ and mixing with $600 \mu \mathrm{L}$ of $43 \mathrm{mmol} / \mathrm{L}$ solution of $\mathrm{H}_{2} \mathrm{O}_{2}$. butylated hydroxy toluene (BHT, $20 \mu \mathrm{g}$ ) was used as a standard and the stock solution was prepared in the same buffer. The absorbance value $(230 \mathrm{~nm})$ of the reaction mixture was recorded at $10 \mathrm{~min}$ intervals between 0 and $40 \mathrm{~min}$. For each concentration, a blank sample was used for background subtraction. The percentage of inhibition was calculated by comparing the results of the test 
with those of the control using the formula: inhibition $(\%)=\left(A_{\mathrm{C}}\right.$ - $\left.A_{\mathrm{T}}\right) / A_{\mathrm{C}} \times 100$, where $A_{\mathrm{C}}$ is the absorbance of the control sample and $A_{\mathrm{T}}$ is the absorbance of test sample.

\section{Results and discussion}

Before commencing our studies, we took into consideration that commonly used gold precatalysts are either unstable $\left(\mathrm{AuCl}_{3}\right)$ or sparingly soluble $\left(\mathrm{AuCl}\right.$ and $\left.\mathrm{AuBr}_{3}\right)$ in water. Therefore, our attention was focused on the use of chloroauric acid $\left(\mathrm{HAuCl}_{4}\right)$, which happens to be the precursor for most of the commercially available gold salts. A mixture of isatin 1a, creatinine and chloroauric acid (1 mol\%) in water was heated to reflux (Scheme 1). Even though, the starting materials were not completely soluble in water, the pure product $\mathbf{2 a}$ was obtained after $30 \mathrm{~min}$, as confirmed by NMR analysis (Table 1, entry 1). Most importantly, the product was obtained stereoselectively as a single diastereoisomer, which was in sharp agreement to Crook's protocol [26]. As chloroauric acid undergoes hydrolysis in water to form $\mathrm{HCl}$ and subsequently catalyze the whole process, a control experiment with substrate 1a was performed by using a $10 \% \mathrm{HCl}$ solution. After $30 \mathrm{~min}$ at reflux temperature, only $10 \%$ of the product $\mathbf{2 a}$ was formed, as indicated by NMR analysis of the crude product. Screening with other potentially oxophilic catalysts such as $\mathrm{Zn}(\mathrm{OTf})_{2}, \mathrm{Cu}(\mathrm{OTf})_{2}$ and $\mathrm{In}(\mathrm{OTf})_{3}$ under similar conditions did not lead to the formation of the product. Pleased with these initial results, we applied chloroauric acid as a catalyst for the reaction of a range of isatin derivatives. Isatin derivatives possessing substituents with a different electronic nature on the nitrogen as well as on the periphery were tolerated and gave excellent yields of the aldol product 2a-2o (Table 1). All the reactions were performed in completely demineralized water $(\mathrm{pH}=7)$ as slight acidity or basicity of the reaction medium could also influence the stereochemical outcome. A blank reaction without chloroauric acid did not proceed at all. One of the beneficial advantages of our protocol is that products with a bromo or an iodo substituent (2d, 2e and 2o) could also be realized. These could potentially serve as synthetic precursors for organometallic cross-coupling reactions [30]. The structural characterization of all the products was established from their spectral data (FTIR, ${ }^{1} \mathrm{H}$ NMR, ${ }^{13} \mathrm{C}$ NMR and MS) and elemental analyses. As an illustrative example, the IR spectrum of compound 3a exhibited sharp peaks at 1718 and $1689 \mathrm{~cm}^{-1}$ corresponding to the carbonyl stretching of oxindole and creatinine cores, respectively. Broad stretching bands between 3384 and $3314 \mathrm{~cm}^{-1}$ suggested the presence of various amide functionalities. A broad parabolic peak between 3557 and $3444 \mathrm{~cm}^{-1}$ revealed the presence of an

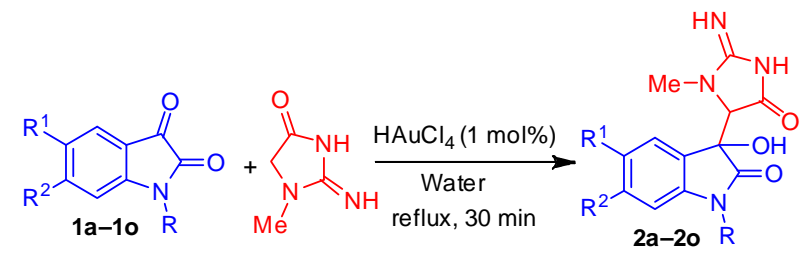

Scheme 1. Au(III)-catalyzed aldolization of isatins with creatinine in water.
Table 1

Synthesis of 3-substituted-3-hydroxyisatins.

\begin{tabular}{lccccc}
\hline Entry & $\mathrm{R}$ & $\mathrm{R}^{1}$ & $\mathrm{R}^{2}$ & Product $^{\mathrm{a}}$ & Yield $^{\mathrm{b}}(\%)$ \\
\hline 1 & $\mathrm{H}$ & $\mathrm{H}$ & $\mathrm{H}$ & $\mathbf{2 a}$ & 95 \\
2 & $\mathrm{H}$ & $\mathrm{F}$ & $\mathrm{H}$ & $\mathbf{2 b}$ & 94 \\
3 & $\mathrm{H}$ & $\mathrm{Cl}$ & $\mathrm{H}$ & $\mathbf{2 c}$ & 94 \\
4 & $\mathrm{H}$ & $\mathrm{Br}$ & $\mathrm{H}$ & $\mathbf{2 d}$ & 95 \\
5 & $\mathrm{H}$ & $\mathrm{I}$ & $\mathrm{H}$ & $\mathbf{2 e}$ & 81 \\
6 & $\mathrm{H}$ & $\mathrm{NO}_{2}$ & $\mathrm{H}$ & $\mathbf{2 f}$ & 90 \\
7 & $\mathrm{Me}$ & $\mathrm{H}$ & $\mathrm{H}$ & $\mathbf{2 g}$ & 96 \\
8 & $\mathrm{Et}$ & $\mathrm{H}$ & $\mathrm{H}$ & $\mathbf{2 h}$ & 97 \\
9 & $\mathrm{Hexyl}$ & $\mathrm{H}$ & $\mathrm{H}$ & $\mathbf{2 i}$ & 95 \\
10 & $\mathrm{Ph}$ & $\mathrm{H}$ & $\mathrm{H}$ & $\mathbf{2 j}$ & 92 \\
11 & $\mathrm{Bn}$ & $\mathrm{H}$ & $\mathrm{H}$ & $\mathbf{2 k}$ & 98 \\
12 & $\mathrm{Ac}$ & $\mathrm{H}$ & $\mathrm{H}$ & $\mathbf{2 l}$ & 91 \\
13 & $\mathrm{Bz}$ & $\mathrm{H}$ & $\mathrm{H}$ & $\mathbf{2 m}$ & 94 \\
14 & $\mathrm{SO}_{2} \mathrm{Ph}$ & $\mathrm{H}$ & $\mathrm{H}$ & $\mathbf{2 n}$ & 92 \\
15 & $\mathrm{H}$ & $\mathrm{Br}$ & $\mathrm{Br}$ & $\mathbf{2 o}$ & 91 \\
\hline
\end{tabular}

all products were characterized by IR, NMR and MS.

${ }^{\mathrm{b}}$ Isolated yield of products after filtration.

intermolecularly bonded hydroxyl group. The ${ }^{1} \mathrm{H}$ NMR spectrum recorded in DMSO- $d_{6}$ showed a singlet at $\delta=4.04 \mathrm{ppm}$, which corresponded to the 4'-methyne hydrogen and a broad singlet at $6.31 \mathrm{ppm}$, which indicated the presence of a hydroxyl group, which was $\mathrm{D}_{2} \mathrm{O}$ exchangeable. In the ${ }^{13} \mathrm{C}$ NMR spectrum, peaks at 175.7 and $182.3 \mathrm{ppm}$ indicated the presence of the carbonyl carbons of isatin and creatinine, respectively. Additionally, the peaks at 69.4 and 76.3 ppm were assigned to the tertiary aliphatic carbon and quaternary carbon (the carbons of the bond between the isatin and creatinine moieties), respectively. Finally, the mass spectrum exhibited a molecular ion peak at $m / z=261[\mathrm{M}+\mathrm{H}]^{+}$, which strongly supported the formation of product $\mathbf{2 a}$. The exclusive diastereoselectivity (an equimolar mixture of $S S$ and $R R$ isomers) of 2 a can be explained by the Zimmerman-Traxler model, which favors anti-selective products over the syn-isomers [31].

Up to this point, we have only discussed the aldolization chemistry of monosubstituted systems. We also used our methodology for the synthesis of more complex systems. As depicted in Scheme 2, we applied our gold(III)-catalyzed procedure for the synthesis of a complex bis-isatinyl system $\mathbf{1 p}$. Thus, treatment of 1,1'-(propane-1,3-diyl)bis-isatin (1.0 mmol) with creatinine $(2.3 \mathrm{mmol})$ under our optimized conditions

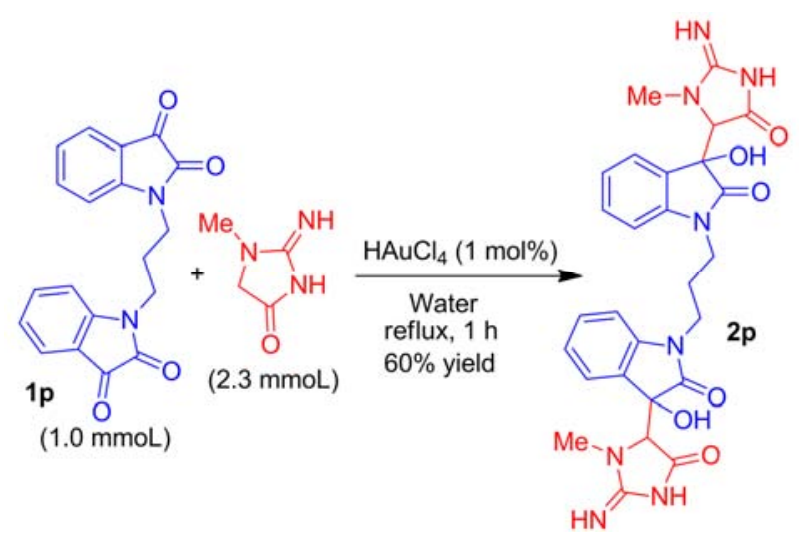

Scheme 2. Au(III)-catalyzed aldolization of bis-isatin 1 p with creatinine in water. 


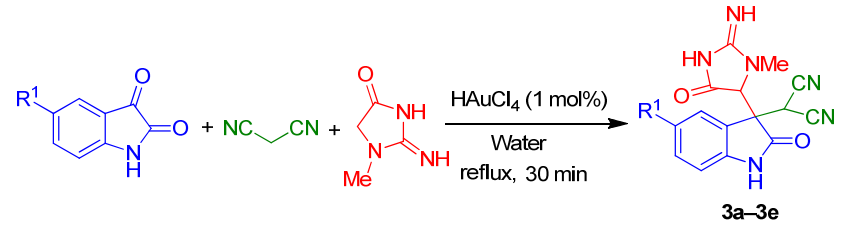

Scheme 3. $\mathrm{Au}(\mathrm{III})$-catalyzed multi-component reaction of isatins, malononitrile and creatinine.

Table 2

Synthesis of adducts $\mathbf{3 a - 3 e .}$

\begin{tabular}{lccc}
\hline Entry & $\mathrm{R}^{1}$ & Product $^{\mathrm{a}}$ & Yield $^{\mathrm{b}}(\%)$ \\
\hline 1 & $\mathrm{H}$ & 3a & 87 \\
2 & $\mathrm{~F}$ & 3b & 90 \\
3 & $\mathrm{Cl}$ & $\mathbf{3 c}$ & 88 \\
4 & $\mathrm{NO}_{2}$ & $\mathbf{3 d}$ & 91 \\
5 & $\mathrm{Me}$ & $\mathbf{3 e}$ & 83 \\
\hline
\end{tabular}

a All products were characterized by IR, NMR and MS.

${ }^{\mathrm{b}}$ Isolated yield of products after filtration.

produced the corresponding bis-aldol product 2 in $60 \%$ yield after reflux for $1 \mathrm{~h}$.

In an attempt to display the synthetic embellishment of our chemistry, we probed the reaction of several isatins with malononitrile and creatinine to realize a sequential condensation/Michael addition (Scheme 3). The reaction resulted in the formation of multicomponent adducts $\mathbf{3 a}-\mathbf{3 e}$ in satisfactory yields (Table 2).

All products were thoroughly characterized by performing spectroscopic analysis. For example, the ${ }^{1} \mathrm{H}$ NMR spectrum of compound 3c exhibited singlets at $\delta=5.98$ and $4.39 \mathrm{ppm}$, which correspond to the $\mathrm{CH}$-proton of the $\mathrm{CH}(\mathrm{CN})_{2}$ group and creatinine moieties, respectively. In the ${ }^{13} \mathrm{C}$ NMR spectrum, the peaks at $\delta=43.9$ and $66.1 \mathrm{ppm}$ corresponded to the carbon flanked by the geminal cyano groups and the tertiary carbon of the creatinine core, respectively. In the mass spectrum, two $\mathrm{Cl}$ isotope peaks at $m / z=343[\mathrm{M}+\mathrm{H}]+$ and $345[\mathrm{M}+\mathrm{H}]^{2+}$ confirmed the formation of product 3c. To establish the stereochemistry of the products, a single crystal of $\mathbf{3 e}$ was obtained and used for $\mathrm{X}$-ray diffraction. Analysis of the crystal structure revealed that

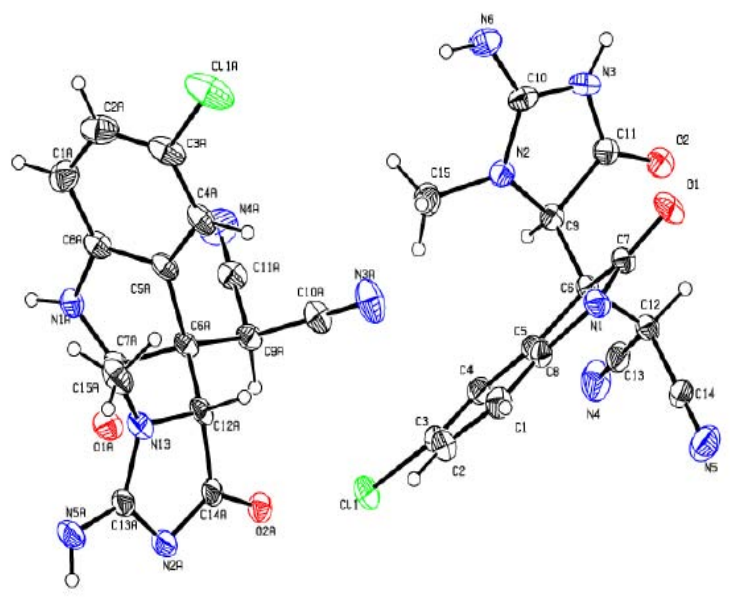

Fig. 2. ORTEP diagram of the X-ray crystal structure of compound 3c.

compound 3e was formed as the anti-isomer ( $R, R$ and $S, S$ enantiomeric pairs) and no syn-stereochemistry was observed (Fig. 2). The exclusive formation of the anti-isomer over syn-isomer was in contrast to our aldolization study.

Because of our continuing interest in chemical biology [32-48] coupled with the information about radical scavenging properties of isatin derivatives [49], we investigated the antioxidant potency of the isatin derivatives 2a-2o by DPPH radical scavenging and hydrogen peroxide methods. The radical scavenging activity [50] of 3-hydroxy isatins was determined spectrophotometrically [51] by using Blois's protocol [52]. All compounds were tested for their interaction with stable free radical DPPH, which specifies their radical scavenging activity. The percentage of inhibition was compared with that of standard L-ascorbic acid (Table 3). The results as a percentage (\%) are expressed as the ratio of absorbance decrease at $517 \mathrm{~nm}$ and the absorbance of DPPH solution in the absence of compounds. A lower absorbance of the reaction mixture indicated higher free radical scavenging activity. The capability to scavenge the DPPH radical was calculated using the equation: DPPH

Table 3

Radical scavenging activity of 3-hydroxyisatins (2a-2o) by the DPPH method.

\begin{tabular}{|c|c|c|c|c|c|c|c|}
\hline \multirow{2}{*}{ No. } & \multirow{2}{*}{ Sample } & \multicolumn{2}{|c|}{ At $50 \mu \mathrm{g} / \mathrm{mL}$} & \multicolumn{2}{|c|}{ At $500 \mu \mathrm{g} / \mathrm{mL}$} & \multicolumn{2}{|c|}{ At $1000 \mu \mathrm{g} / \mathrm{mL}$} \\
\hline & & Absorbance & Activity (\%) & Absorbance & Activity (\%) & Absorbance & Activity (\%) \\
\hline 1 & L-Ascorbic acid & 0.035 & 96 & 0.026 & 97 & 0.008 & 99 \\
\hline 2 & $2 a$ & 0.721 & 19 & 0.329 & 63 & 0.213 & 76 \\
\hline 3 & $2 \mathbf{b}$ & 0.704 & 21 & 0.427 & 52 & 0.276 & 69 \\
\hline 4 & $2 c$ & 0.721 & 19 & 0.302 & 66 & 0.133 & 85 \\
\hline 5 & $2 d$ & 0.739 & 17 & 0.463 & 48 & 0.178 & 80 \\
\hline 6 & $2 e$ & 0.704 & 21 & 0.311 & 65 & 0.115 & 87 \\
\hline 8 & $2 g$ & 0.650 & 27 & 0.320 & 64 & 0.267 & 70 \\
\hline 9 & $2 \mathrm{~h}$ & 0.802 & 10 & 0.686 & 23 & 0.516 & 42 \\
\hline 10 & $2 \mathbf{i}$ & 0.757 & 15 & 0.516 & 42 & 0.240 & 73 \\
\hline 11 & $2 \mathbf{j}$ & 0.695 & 22 & 0.401 & 55 & 0.222 & 75 \\
\hline 12 & $2 \mathbf{k}$ & 0.873 & 02 & 0.784 & 12 & 0.579 & 35 \\
\hline 13 & 21 & 0.666 & 26 & 0.375 & 58 & 0.219 & 75 \\
\hline 14 & $2 \mathrm{~m}$ & 0.720 & 19 & 0.330 & 64 & 0.215 & 77 \\
\hline
\end{tabular}


Table 4

Radical scavenging activity of 3-hydroxyisatins (2a-2o) by the $\mathrm{H}_{2} \mathrm{O}_{2}$ method.

\begin{tabular}{|c|c|c|c|c|c|c|c|c|c|c|c|}
\hline \multirow[b]{2}{*}{ No. } & \multirow[b]{2}{*}{ Sample } & \multicolumn{2}{|c|}{ At $0 \mathrm{~min}$} & \multicolumn{2}{|c|}{ At $10 \mathrm{~min}$} & \multicolumn{2}{|c|}{ At $20 \mathrm{~min}$} & \multicolumn{2}{|c|}{ At $30 \mathrm{~min}$} & \multicolumn{2}{|c|}{ At $40 \mathrm{~min}$} \\
\hline & & Absorbance & $\begin{array}{c}\text { Activity } \\
(\%)\end{array}$ & Absorbance & $\begin{array}{c}\text { Activity } \\
(\%)\end{array}$ & Absorbance & $\begin{array}{c}\text { Activity } \\
(\%)\end{array}$ & Absorbance & $\begin{array}{c}\text { Activity } \\
(\%)\end{array}$ & Absorbance & $\begin{array}{c}\text { Activity } \\
(\%)\end{array}$ \\
\hline 1 & BHT & 0.072 & 92 & 0.126 & 86 & 0.153 & 83 & 0.162 & 82 & 0.180 & 80 \\
\hline 2 & $2 \mathbf{a}$ & 0.514 & 43 & 0.586 & 35 & 0.712 & 21 & 0.799 & 20 & 0.757 & 16 \\
\hline 3 & $2 b$ & 0.126 & 86 & 0.135 & 85 & 0.234 & 74 & 0.252 & 72 & 0.279 & 69 \\
\hline 4 & $2 c$ & 0.108 & 88 & 0.135 & 85 & 0.216 & 76 & 0.243 & 73 & 0.270 & 70 \\
\hline 5 & $2 d$ & 0.063 & 93 & 0.144 & 84 & 0.162 & 82 & 0.207 & 77 & 0.261 & 71 \\
\hline 7 & $2 f$ & 0.144 & 84 & 0.153 & 83 & 0.180 & 80 & 0.243 & 73 & 0.288 & 68 \\
\hline 8 & $2 \mathrm{~g}$ & 0.162 & 82 & 0.171 & 81 & 0.234 & 74 & 0.261 & 71 & 0.315 & 65 \\
\hline 9 & $2 h$ & 0.387 & 57 & 0.514 & 43 & 0.550 & 39 & 0.631 & 30 & 0.712 & 21 \\
\hline 10 & $2 \mathbf{i}$ & 0.135 & 85 & 0.180 & 80 & 0.162 & 82 & 0.225 & 75 & 0.315 & 65 \\
\hline 11 & $2 \mathbf{j}$ & 0.117 & 87 & 0.153 & 83 & 0.225 & 75 & 0.270 & 70 & 0.369 & 59 \\
\hline 12 & $2 k$ & 0.360 & 60 & 0.487 & 45 & 0.541 & 45 & 0.586 & 35 & 0.685 & 24 \\
\hline 13 & 21 & 0.145 & 84 & 0.150 & 84 & 0.189 & 81 & 0.245 & 72 & 0.295 & 67 \\
\hline 16 & Control & & & & & 0.9022 & & & & & \\
\hline
\end{tabular}

scavenging effect $(\%)=\left(A_{\mathrm{c}}-A_{\mathrm{t}} / A_{\mathrm{c}}\right) \times 100$, where $A_{\mathrm{c}}$ is the absorbance of the control reaction and $A_{\mathrm{t}}$ is the absorbance in the presence of samples or standards. Analysis of the screening results revealed that the radical scavenging activity of the compounds on DPPH radicals increases with respect to the concentration (Table 3). Compounds possessing 5-Cl (2c), 5-Br (2d) and 5-I (2e) moieties showed maximum activity at a concentration of $1000 \mu \mathrm{g} / \mathrm{mL}$. This could be attributed to the better homolysis tendency of the carbon-halide bond leading to the respective free radicals. The radical scavenging activity of compounds possessing $\mathrm{N}-\mathrm{Et}(\mathbf{2} \mathbf{h})$ and $\mathrm{N}-\mathrm{Bn}(\mathbf{2 k})$ groups was less potent compared with the standard. Hydrogen peroxide radical scavenging activity [50] was performed using a solution of hydroxyisatins $\mathbf{2 a - 2 o}$ in a mixture of phosphate buffer and a solution of $\mathrm{H}_{2} \mathrm{O}_{2}$ by a spectrophotometric method and compared with the standard BHT. The absorbance value $(230 \mathrm{~nm})$ of the reaction mixture was recorded at 10 min intervals between 0 and $40 \mathrm{~min}$. For each concentration, a blank sample was used for background subtraction and the corresponding absorbance value for $\mathbf{2 a - 2 o}$ is given in Table 4 . The compound containing a 5-iodo group (2e) exhibited maximum antioxidant potential after $40 \mathrm{~min}$. Compounds $\mathbf{2 d}$ and $\mathbf{2 c}$ emerged as the second most active compounds among those tested, with an absorbance value of $71 \%$ and $70 \%$, respectively. This observation was in agreement with the results obtained using the DPPH protocol (compare Table 3). However, the antioxidant properties of the compounds possessing N-Et (2h) and N-Bn (2k) and no substitution (2a) were poor, as evidenced by their low absorbance values. The other compounds screened in this study exhibited inhibition that was comparable with the standard, BHT.

\section{Conclusions}

In summary, we prepared of 3-hydroxy-3-(2-imino-3-methyl-5-oxoimidazolidin-4-yl)indolin-2-one derivatives in excellent yields as single diastereoisomers (syn-selectivity) through the chloroauric acid catalyzed aldolization between isatins and creatinine under aqueous conditions. The reaction was facile, high-yielding, and did not require solvent extraction and column chromatography. The radical scavenging activity of the prepared 3-hydroxyisatin derivatives revealed that compounds possessing halo groups at the C5-carbon (2c, 2d and 2c) exhibited comparable antioxidant potency to the standard. Furthermore, we applied our reaction conditions to a tandem reaction between isatin, creatinine and malononitrile. In this case, the products were obtained in excellent yield with complete an$t i$-selectivity. Further studies aimed towards the preparation of more complex systems as well as kinetic studies to investigate the reaction mechanism are currently underway in our laboratory and will be reported in due course.

\section{Acknowledgments}

K. P thanks the Central Council for Research in Siddha (CCRS), India and T. P thanks the management of Inogent Laboratories Ltd., India. C. P. is grateful to the Science and Engineering Research Board (SERB) for the financial support (SERB/F/5666/2015-16).

\section{References}

[1] J. E. DeLorbe, D. Home, R. Jove, S. M. Mennen, S. Nam, F. L. Zhang, L. E. Overman, J. Am. Chem. Soc., 2013, 135, 4117-4128.

[2] L. Liu, S. L. Zhang, F. Xue, G. S. Lou, H. Y. Zhang, S. C. Ma, W. H. Duan, W. Wang, Chem. Eur. J., 2011, 17, 7791-7795.

[3] S. Mohammadi, R. Heiran, R. P. Herrera, E. Marqués-López, ChemCatChem, 2013, 5, 2131-2148.

[4] S. Peddibhotla, Curr. Bioact. Compd., 2009, 5, 20-38.

[5] F. Zhou, Y. L. Liu, J. Zhou, Adv. Synth. Catal., 2010, 352, 1381-1407.

[6] J. J. Badillo, N. V. Hanhan, A. K. Franz, Curr. Opin. Drug Discovery Dev., 2010, 13, 758-776.

[7] A. Kumar, S. S. Chimni, RSC Adv., 2012, 2, 9748-9762.

[8] O. Riant, J. Hannedouche, Org. Biomol. Chem., 2007, 5, 873-888.

[9] M. Bella, T. Gasperi, Synthesis, 2009, 1583-1614.

[10] U. M. Lindstörm, Chem. Rev., 2002, 102, 2751-2772. 


\section{Graphical Abstract}

Chin. J. Catal., 2017, 38: 775-783 doi: 10.1016/S1872-2067(17)62812-2

Gold-catalyzed addition reaction between creatinine and isatin: A sustainable and green chemistry approach for the diastereoselective synthesis of 3-substituted-3-hydroxyisatins

K. Parthasarathy, T. Ponpandian, C. Praveen*

Siddha Central Research Institute, Central Council for Research in Siddha (CCRS), India; Inogent Laboratories Private Limited, India;

CSIR-Central Electrochemical Research Institute (CSIR-CECRI), India<smiles>[R16]c1ccc2c(c1)C(=O)C(=[O+][O-])N2c1ccc2c(c1)C(=O)C(=O)N2[R]</smiles>

Aldolization and three component condensation of isatin derivatives with creatinine under the catalysis of auric acid in water has been developed. The synthesized compounds exhibited moderate radical scavenging activity.

[11] S. Paladhi, M. Bhati, D. Panda, J. Dash, J. Org. Chem., 2014, 79, 1473-1480.

[12] S. Paladhi, A. Chauhan, K. Dhara, A. K. Tiwari, J. Dash, Green Chem., 2012, 14, 2990.

[13] C. Praveen, P. T. Perumal, Chin. J. Catal., 2016, 37, 288-299.

[14] C. Praveen, P. T. Perumal, Synthesis, 2016, 48, 855-864.

[15] C. Praveen, K. H. Kumar, D. Muralidharan, P. T. Perumal, Tetrahedron, 2008, 64, 2369-2374.

[16] C. Praveen, S. Jegatheesan, P. T. Perumal, Synlett, 2009, 2795-2800.

[17] C. Praveen, P. Kiruthiga, P. T. Perumal, Synlett, 2009, 1990-1996.

[18] C. Praveen, K. Karthikeyan, P. T. Perumal, Tetrahedron, 2009, 65, 9244-9255.

[19] C. Praveen, Y. W. Sagayaraj, P. T. Perumal, Tetrahedron Lett., 2009, 50, 644-647.

[20] C. Praveen, C. Iyyappan, P. T. Perumal, Tetrahedron Lett., 2010, 51, 4767-4771.

[21] C. Praveen, P. T. Perumal, Synlett, 2011, 521-524.

[22] B. Balakrishnan, C. Praveen, P. R. Seshadri, P. T. Perumal, Acta Cryst. E, 2011, 67, 01575-U1900.

[23] B. Balakrishnan, C. Praveen, P. R. Seshadri, P. T. Perumal, Acta Cryst. E, 2013, 69, 0597-0597

[24] C. Praveen, K. Parthasarathy, P. T. Perumal, Synlett, 2010, $1635-1640$.

[25] A. Skiredj, M. A. Beniddir, D. Joseph, G. Bernadat, L. Evanno, E. Poupon, Synthesis, 2016, 47, 2367-2376.

[26] N. R. Penthala, T. R. Yerramreddy, N. R. Madadi, P. A. Crooks, Bioorg. Med. Chem. Lett., 2016, 20, 4468-4471.

[27] N. R. Penthala, T. R. Y. Reddy, S. Parkin, P. A. Crooks, Acta Cryst. E, 2009, 65, 02909-02910.

[28] N. R. Penthala, T. R. Yerramreddy, S. Parkin, P. A. Crooks, Acta Cryst. E, 2009, 65, 02439-02440.

[29] N. R. Penthala, T. R. Yerramreddy, S. Parkin, P. A. Crooks, Acta Cryst. E, 2009, 65, 0552/1-0522/7.

[30] K. C. Nicolaou, P. G. Bulger, D. Sarlah, Angew. Chem. Int. Ed., 2005,
$44,4442-4489$.

[31] H. E. Zimmerman, M. D. Traxler, J. Am. Chem. Soc., 1957, 79, 1920-1923.

[32] J. C. Jeyaveeran, C. Praveen, Y. Arun, A. A. M. Prince, P. T. Perumal, J. Chem. Sci., 2016, 128, 73-83.

[33] J. C. Jeyaveeran, C. Praveen, Y. Arun, A. A. M. Prince, P. T. Perumal, J. Chem Sci., 2016, 128, 787-802.

[34] C. Praveen, D. B. Ananth, Bioorg. Med. Chem. Lett., 2016, 26, 2507-2512.

[35] C. Praveen, A. Ayyanar, P. T. Perumal, Bioorg. Med. Chem. Lett., 2011, 21, 4170-4173.

[36] C. Praveen, C. Iyyappan, K. Girija, K. S. Kumar, P. T. Perumal, J. Chem. Sci., 2012, 124, 451-462.

[37] C. Praveen, A. Nandakumar, P. Dheenkumar, D. Muralidharan, P. T. Perumal, J. Chem. Sci., 2012, 124, 609-624.

[38] C. Praveen, P. Dheenkumar, P. T. Perumal, J. Chem. Sci., 2013, 125, 71-83.

[39] C. Praveen, K. Parthasarathy, P. S. Kumar, P. T. Perumal, Indian. J. Chem. Sect. B, 2015, 54B, 373-382.

[40] C. Praveen, C. Iyyappan, P. T. Perumal, K. Girija, K. Indian. J. Chem. Sect. B, 2012, 51B, 498-507.

[41] K. Parthasarathy, C. Praveen, K. Saranraj, C. Balachandran, P. S. Kumar, Med. Chem. Res., 2016, 25, 2155-2170.

[42] K. Parthasarathy, C. Praveen, J. C. Jeyaveeran, A. A. M. Prince, Bioorg. Med. Chem. Lett., 2016, 26, 4310-4317.

[43] K. Parthasarathy, C. Praveen, C. Balachandran, P. S. Kumar, S. Ignacimuthu, P. T. Perumal, Bioorg. Med. Chem. Lett., 2013, 23, 2708-2713.

[44] C. Praveen, S. Narendiran, P. Dheenkumar, P. T. Perumal, J. Chem. Sci., 2013, 125, 1543-1553.

[45] C. Praveen, A. Ayyanar, P. T. Perumal, Bioorg. Med. Chem. Lett., 2011, 21, 4072-4077.

[46] K. Parthasarathy, C. Praveen, P. S. Kumar, C. Balachandran, P. T. Perumal, RSC Adv., 2015, 5, 15818-15830.

[47] C. Praveen, P. DheenKumar, D. Muralidharan, P. T. Perumal, 
Bioorg. Med. Chem. Lett., 2010, 20, 7292-7296.

[48] C. Praveen, A. Kalyanasundaram, P. T. Perumal, Synlett, 2010, 777-781.

[49] C. R. Prakash, S. Raja, G. Saravanan, P. D. Kumar, P. T. Selvam, Asian J. Res. Pharm. Sci., 2011, 1, 140-143.
[50] J. R. Soares, T. C. P. Dinis, A. P. Cunha, L. M. Ameida, Free Radical Res., 1997, 26, 469-478.

[51] T. Hatano, H. Kagawa, T. Yasuhara, T. Okuda, Chem. Pharm. Bull., 1988, 36, 2090-2097.

[52] M. S. Blois, Nature, 1958, 26, 1199-1200.

\title{
金催化肌酸酐与靛红的加成反应: 一种可持续的绿色方法用于非对映选择合成 3-取代的3-羟基靛红
}

\author{
K. Parthasarathy ${ }^{\mathrm{a}}$, T. Ponpandian ${ }^{\mathrm{b}}$, C. Praveen ${ }^{\mathrm{c}, *}$ \\ a 悉达中央研究委员会 悉达中央研究所化学系, Arumbakkam, 金奈600106, 泰米尔纳德邦, 印度 \\ $\mathrm{b}$ 工业发展区私人有限实验室, 海得拉巴500076, 特伦甘纳邦, 印度 \\ c 中央电化学研究所功能材料部科学和工业研究理事会, 卡拉库迪 600006 , 泰米尔纳德邦, 印度
}

摘要: 报道了水介质中金催化肌酸䣶与不同靛红之间的醛醇缩合反应. 该法无需繁杂的溶剂萃取和柱色谱技术, 只需简单 的过滤即可将产物分离出来, 因而操作简单. 通过较宽范围的靛红衍生物的反应, 均可高产率并完全地制取相应的顺式醛 醇缩合产物, 因此该法表现出较高的通用性. 还将该合成策略进一步拓展至靛红, 肌酸酐和丙二腈的串联反应, 可高产率、 完全的制取反式多组分产物. 采用分光光度法测定了合成产物的抗氧化性能, 结果表明, 与标准物相比,含有卤素原子的三 个化合物(2c, 2d和 $2 e)$ 表现出最高的活性.

关键词: 肌酸酐; 金催化; 绿色化学; 非对映立体选择性; 抗氧化剂

收稿日期: 2017-01-05. 接受日期: 2017-02-28. 出版日期: 2017-05-05.

*通讯联系人. 电话: $+91-9677733808$; 电子信箱: chandrasekar.praveen@gmail.com

本文的英文电子版由Elsevier出版社在ScienceDirect上出版(http://www.sciencedirect.com/science/journal/18722067). 